\title{
Cerebral hemorrhagic infarction as the initial manifestation of deep venous thrombosis in a child with patent foramen ovale
}

${ }^{1}$ Pediatric Hospital of Athens, "Aglaia Kyriakou”, Neurosurgical Department, Athens, Greece

${ }^{2}$ Cardiology Department, Pediatric hospital “Agia Sophia”, Athens, Greece 3 Hemodynamic Cardiology

Department, Pediatric hospital “Agia Sophia”, Athens, Greece

^Email: dimpanayop@gmail.com http://dx.doi.org/

10.21542/gcsp.2018.17

Received: 22 October 2017

Accepted: 18 April 2018 (C) 2018 The Author(s), licensee Magdi Yacoub Institute. This is an open access article distributed under the terms of the Creative Commons Attribution license CC BY-4.0, which permits unrestricted use, distribution and reproduction in any medium, provided the original work is properly cited.
Dimitrios Panagopoulos ${ }^{1 *}$, Sofia Loukopoulou², Evagelos Karanasios², Georgia Grigoriadou ${ }^{1}$, Nikolaos Eleftherakis 3

\section{ABSTRACT}

Arterial ischemic stroke (AIS), with an estimated incidence of 1.1-4.3 per 100,000, is an important cause of morbidity and mortality in children and the risk of recurrence is high. We present the case of an 11-year-old child who presented with a symptomatology of acute ischemic stroke of unknown etiology. The radiological investigation did not reveal any underlying brain abnormality that could cause the event. The diagnostic work up included an echocardiogram, which revealed a thrombus in the right atrium, in conjunction with a patent foramen ovale. The patient was initiated immediately on anticoagulation therapy with low molecular weight heparin and warfarin, but two days later she suffered pulmonary emboli, diagnosed with spiral thorax computed tomography (CT) scan. An ultrasound study of the vessels of the lower extremities revealed deep venous thrombosis (DVT), which was considered to be the underlying causative mechanism. 


\section{INTRODUCTION}

To the best of our knowledge, this is the first documented case of right atrial thrombus resulting from deep venous thrombosis in a pediatric patient with patent foramen ovale and associated ischemic stroke event.

A discussion regarding the definition of cryptogenic stroke, its etiology and relationship with deep venous thrombosis and the currently proposed therapy, follows.

\section{CASE REPORT}

We present a rare clinical case of a young girl harboring a latent deep venous thrombosis (DVT), a thrombus in the right atrium with subsequent arterial ischemic stroke (AIS) of the brain, possibly due to a patent foramen ovale (PFO). This was the first clinical manifestation of an otherwise unrecognized clinical condition.

An 11-year old girl presented with headache, vomiting, dizziness, dysphasia and gaze dedication for a few seconds. The initial computed tomography (CT) scan revealed intracerebral hematoma $(\mathrm{ICH})$ in the right parieto-occipital region with perilesional edema (Figure 1).

Patient was admitted in the neurosurgical clinic and was initiated on anticonvulsant medication. Neurological and ophthalmological examination did not reveal any focal deficits. MRI performed the same day revealed edematous configuration of the nearby gyri with concurrent presentation of hemorrhagic elements (Figure 2).

An electroencephalography (EEG) study detected focal cerebral disturbances. A repeat MRI scan (with contrast) and MR angiography (MRA), indicated a hemorrhagic infarct in the territory in a subacute phase with related edema (hemorrhagic stroke). MRA further recognized stenosis of the right (middle cerebral artery) MCA with obstruction of the posterior peripheral branches (Figure 3).

Consequently, a digital subtraction angiography (DSA) from the femoral artery was performed which did not reveal underlying vascular abnormalities (Figure 4).

A thorough investigation for hypercoagulable states (deficiencies of protein $C$ and anti III, protein S, antithrombin and plasminogen, molecular studies for factor $\mathrm{V}$ Leiden, prothrombin 20210A, homocysteine, MTHFR gene mutations) and immunologicalrheumatological conditions (HLA-51, C-ANCA, p-ANCA, anti-GBM, LA1 and LA2, | $\beta$-2GPI, ACA IgM and IgG antibodies) did not reveal any abnormalities.

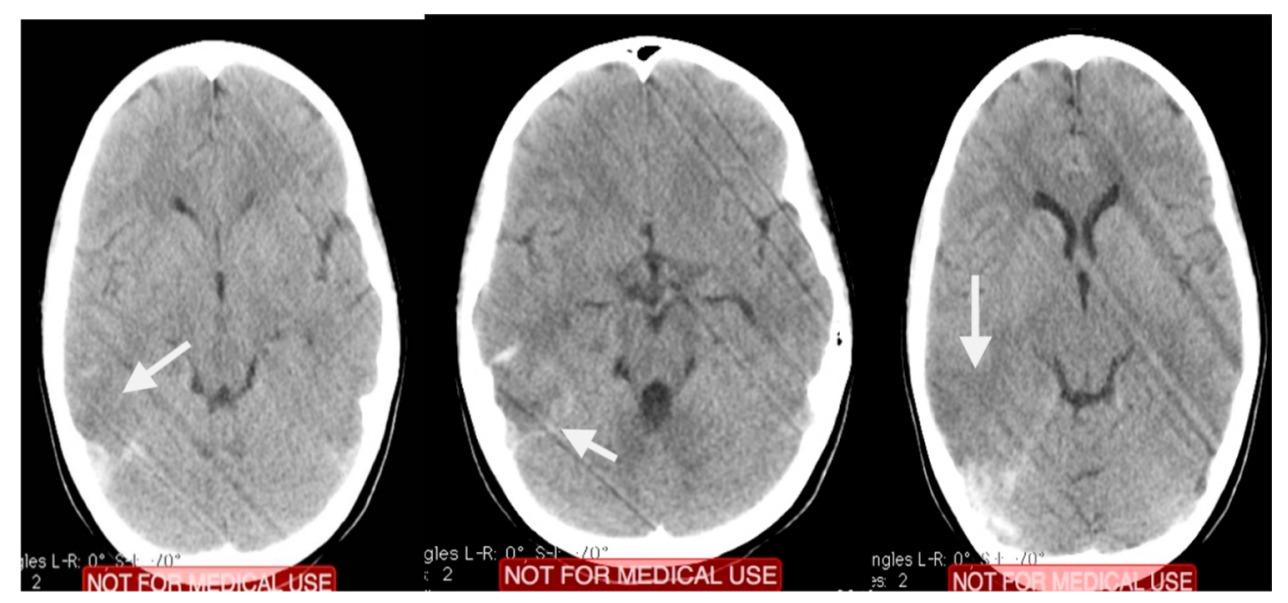

Figure 1. Initial CT scan, after the ictus. Arrows depict the areas of ischemic stroke (hypodense), with a surrounding area of hemorrhagic transformation. 


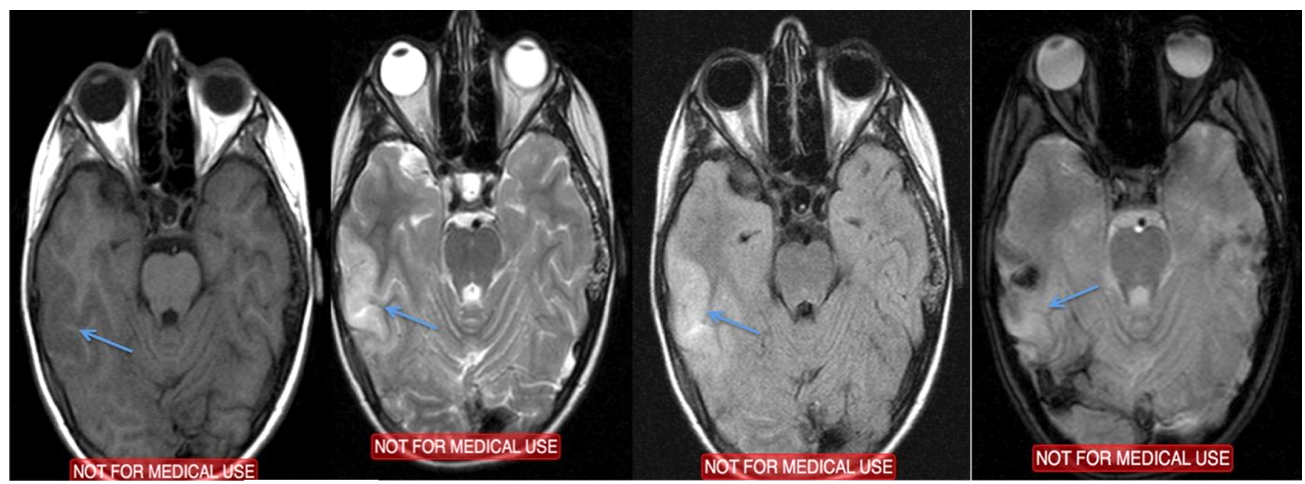

Figure 2. Initial MRI scan within one day after the event. Arrows depict the pathologic changes at T1W, T2W, FLAIR and T2 GRE sequences.

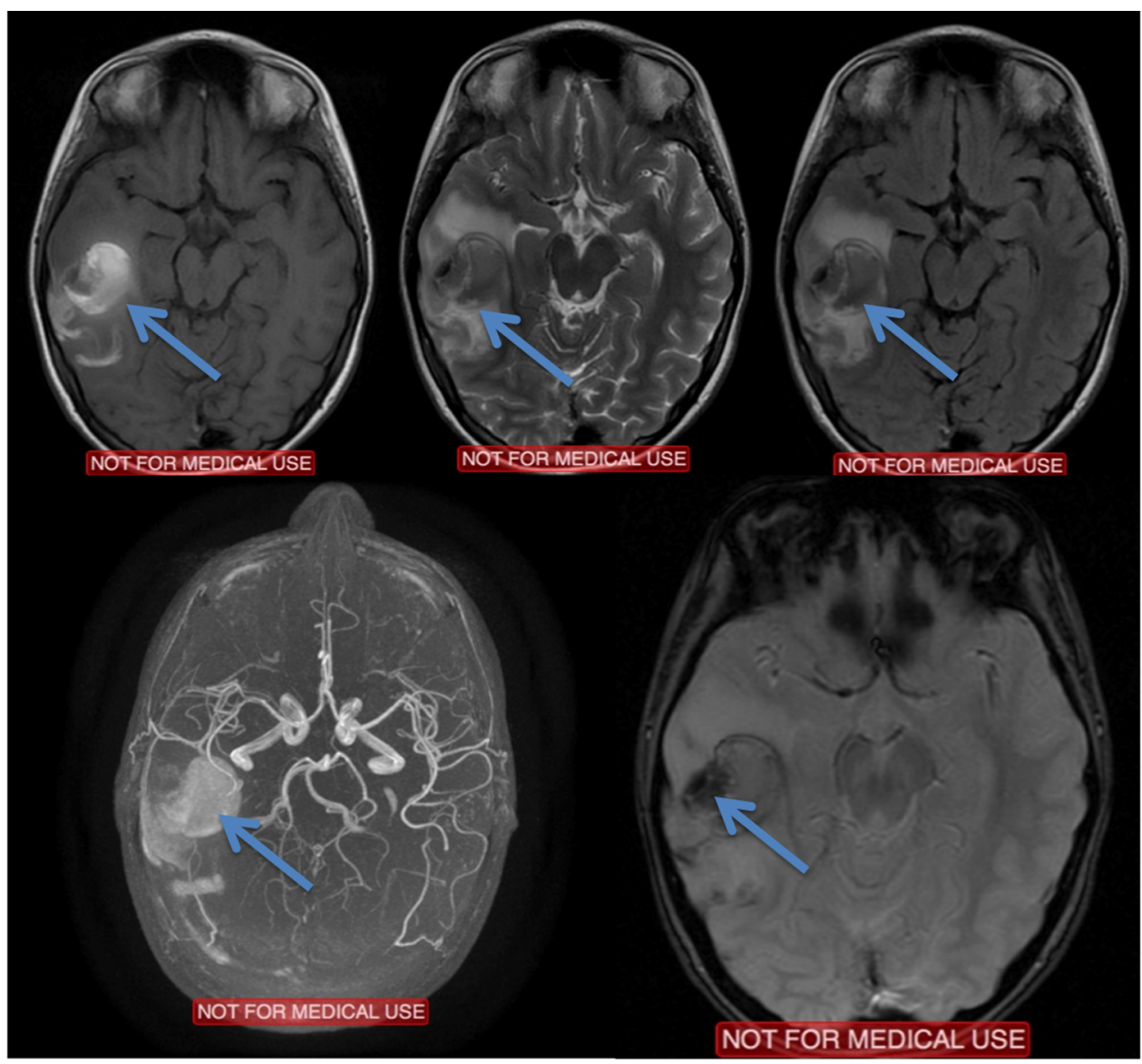

Figure 3. Arrows depict the evolution of signal changes, 10 days after the event. MRI and MRA scan after 10 days. T1W, T2W, FLAIR and T2-GRE images and MRA reconstruction.

An ultrasound study of the vessels of the lower extremities revealed an intraluminal thrombus of the left superficial femoral and popliteal vein. A thoroughly detailed investigation of patient's history revealed a minor sports related blunt injury of the left lower extremity a day before the initial symptoms, which was associated with lower extremities ultrasound findings. Additionally, an echocardiogram visualized a thrombus $(2 \times 1,5 \mathrm{~cm})$ attached to the right atrium in conjunction with patent foramen ovale (Figures 5 and 6). 


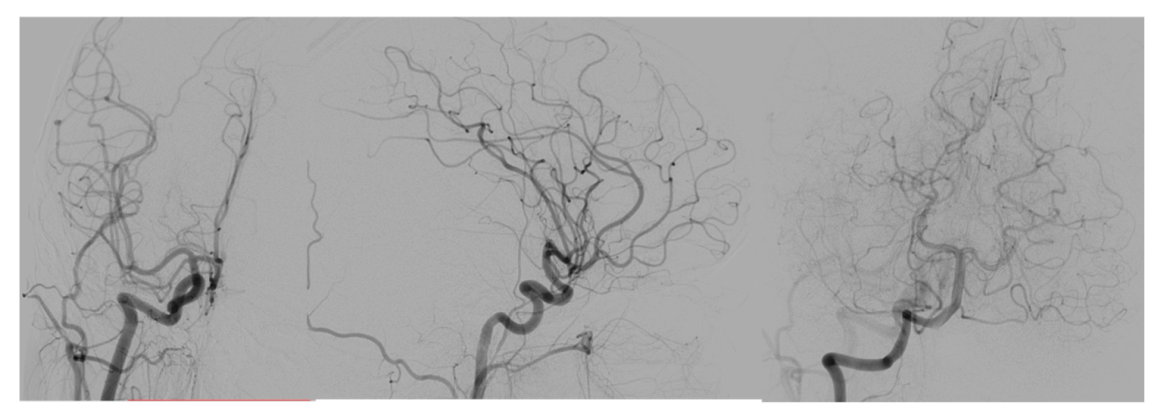

Figure 4. Digital subtraction angiography (DSA) imaging, revealing no pathological findings.

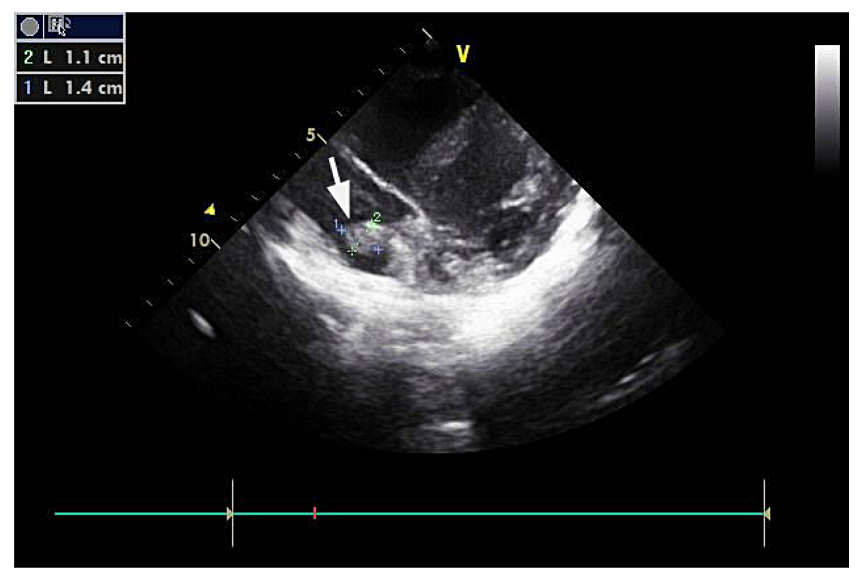

Figure 5. Arrows in Figures 5 and 6 indicate the location of the thrombus with its approximate dimensions.

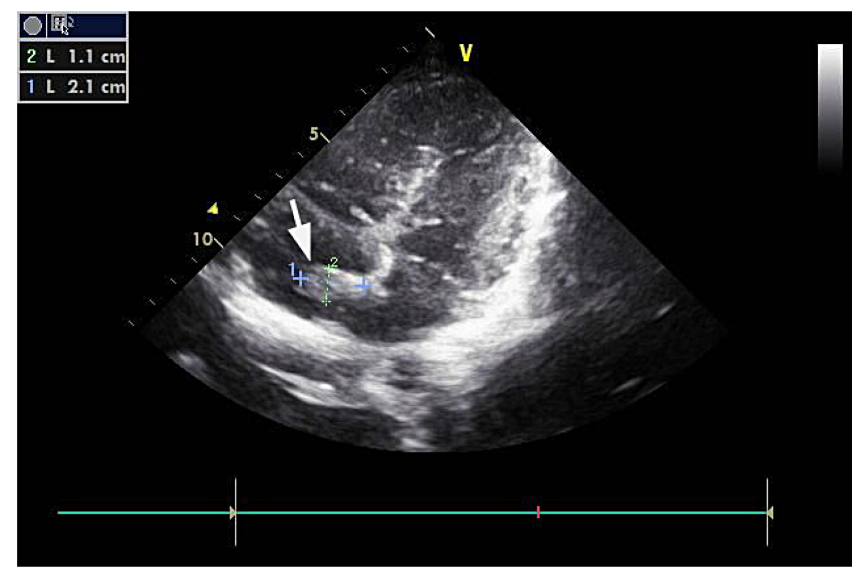

Figure 6. Arrows in Figures 5 and 6 indicate the location of the thrombus with its approximate dimensions.

Due to the relative contraindication for thrombolysis, patient was initiated immediately on anticoagulation therapy with low molecular weight heparin and warfarin. Two days later, she developed acute symptoms of dyspnea and chest pain and a subsequent spiral thorax CT revealed pulmonary emboli at the left pulmonary artery, as long as the persistence of the atrial thrombus. Anticoagulation therapy was continued and a foramen ovale umbrella placement was later performed (Figure 7). 


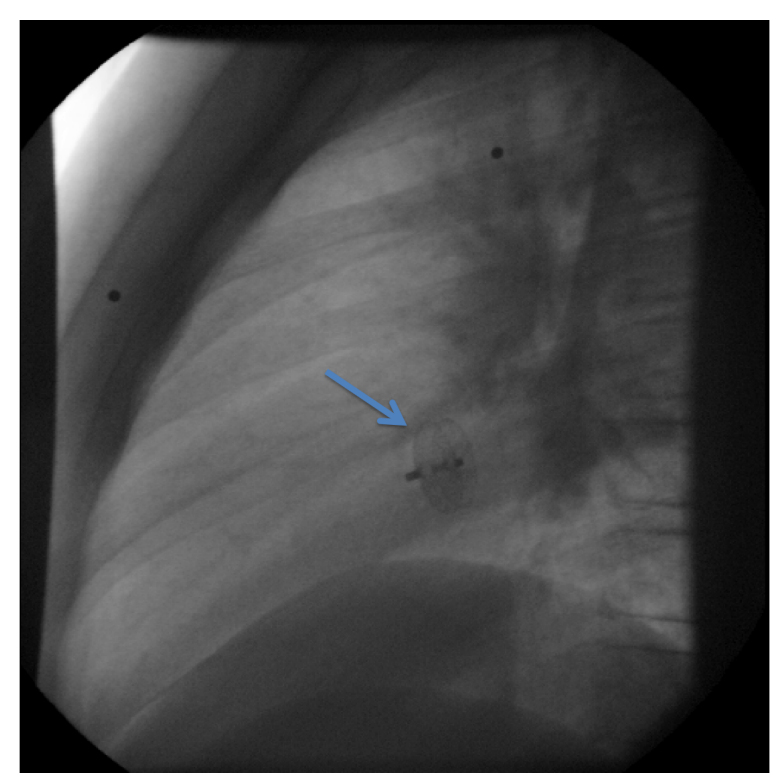

Figure 7. The arrow indicates the site of placement of the umbrella.

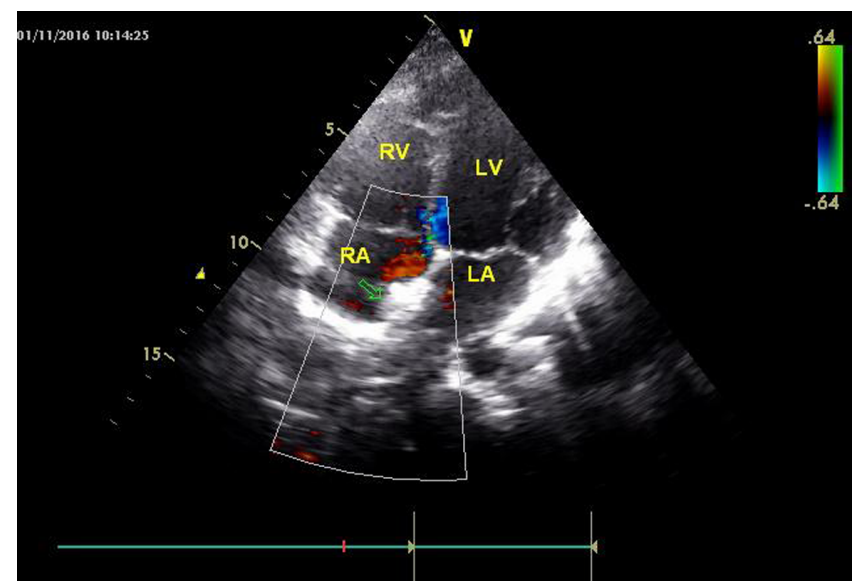

Figure 8. Arrow indicates the position of the umbrella.

Patient remained symptom free in the follow-up period and serial cardiac ultrasound examinations revealed gradual resolution of the right atrial thrombus (Figure 8).

A few months later, a repeat MRI scan was performed, while the patient being neurologically normal. The examination verified the known lesion at the right temporaloccipital lobe region, which revealed characteristics, compatible with a chronic lesion. More specifically, the imaging of the lesion identified a territory with intermediate to hypo-intensity signal at Flair sequences and hemosiderin ring at GRE sequences (Figure 9).

\section{DISCUSSION}

\section{Epidemiology-definition of cryptogenic stroke}

Arterial ischemic stroke (AIS), with an estimated incidence of 1.1-4.3 per 100,000, is an important cause of morbidity and mortality in children and the recurrence risk is high ${ }^{1}$. It 


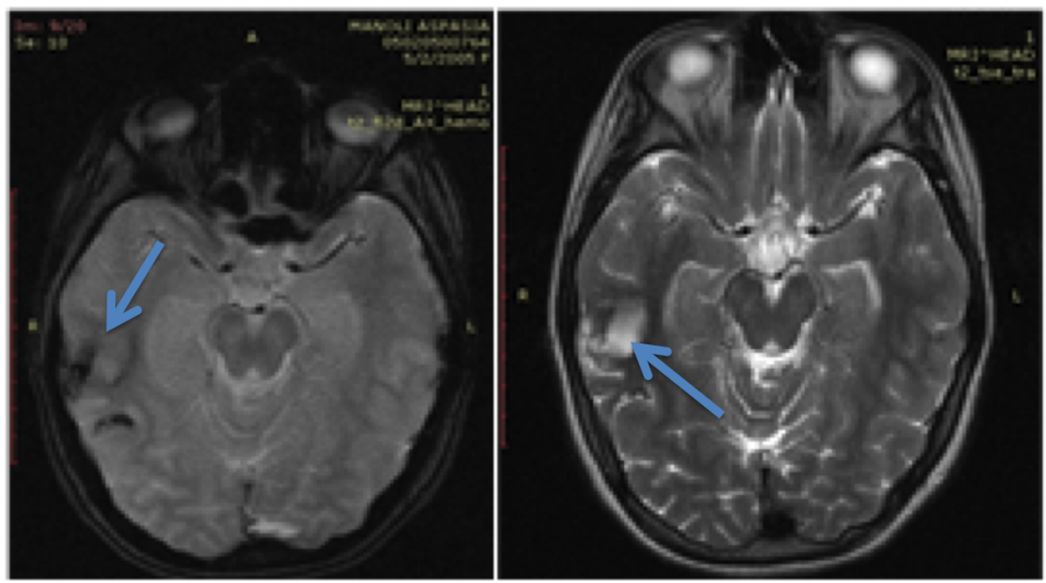

Figure 9. The left image is an MRI GRE sequence, showing the hemosiderin ring (arrow) and the right image is a FLAIR sequence, revealing an area of intermediate to hypointensity signal (arrow).

is defined as an acute clinical syndrome with a neurological deficit referable to a cerebral arterial territory and a brain MRI showing a corresponding area of acute infarct.

A stroke is termed "cryptogenic" when its etiology cannot be attributed to any specific cause after an extensive search for the most common causes, such as atherosclerosis of the intracranial vessels, lacunar damage from hypertension, or embolus derived from a thrombus located in the left atrium, the left ventricular apex, or at the level of an ulcerated plaque of the aortic arch.

\section{Etiology}

The etiology of AIS remains undetermined in a high proportion of children. Predisposing conditions for ischemic cerebrovascular accidents in children include congenital heart malformations (congenital cyanotic complex heart malformations or acquired heart disease), sickle cell disease, infections, and collagen tissue abnormalities ${ }^{2}$, but around half occur in children who were previously well (cryptogenic stroke) 3 . It is well known from the literature, that one of the most common conditions associated with AIS is congenital heart malformations (like patent foramen ovale) 4-14 $^{4}$.

\section{Paradoxic embolism and stroke}

Recently, paradoxical embolism across the PFO was suggested as a possible etiology in some of these children ${ }^{15}$. PFO is reported, as an autopsy finding, to remain patent in about $25 \%$ of adults, thus presenting a potential passageway for paradoxical embolization. On some particular circumstances such as during Valsalva maneuver, which is reproduced by the act of defecating or coughing, the reversal of the physiologic inter-atrial pressure gradient results in right-to-left shunting across the PFO and contributes to the passage of embolic material. The prevalence of PFO was significantly higher in patients with cryptogenic stroke versus those with known causes of stroke ( $42 \%$ vs $7 \%$ ), indicating that PFO is associated with cryptogenic stroke ${ }^{16,17}$. The association is documented in case reports $2,3,18-22$. However, the direct role of a PFO in stroke remains unclear $^{23}$.

In our case, the dual (and simultaneous) detection of thrombi in the deep venous system and the right atrium along with the rapid sequence of embolic events in the absence of other underlying pathological conditions, point out the paradoxical embolism through a patent PFO as the most plausible scenario for the ischemic stroke. 


\section{Prothrombotic disorders}

Also, prothrombotic disorders are frequently identified in pediatric patients with stroke ${ }^{7}$ and case control studies demonstrate an association of arterial ischemic stroke in children with hereditary prothrombotic risk factors 4 . Another study reports prothrombotic abnormalities to be present in $20-50 \%$ of children with arterial ischemic stroke $\mathrm{e}^{11,24}$.

Reasoning the coexistence of deep venous thrombosis and right atrial cavity thrombus in our patient with the absence of positive laboratory results for hypercoagulable disorders, we speculate either a transient hypercoagulable state, possibly associated with patient's minor sport related injury or an unidentified mechanism by our thrombophilia screening.

\section{Deep venous thrombosis and cryptogenic stroke}

Young adults with cryptogenic ischemic stroke are more likely to have both patent foramen ovale and pelvic deep vein thrombosis (DVT) than young adults with ischemic stroke of known cause. Young patients with cryptogenic transient ischemic attack (TIA) or stroke and patent foramen ovale (PFO) should be evaluated for lower-extremity or pelvic venous thrombosis, which would be an indication for anticoagulation. In our case, screening for underlying causes of cryptogenic stroke with ultrasound of the lower extremities revealed venous thrombosis. The most probable releasing factor (and causative) of this event was a few days previously reported, sports related, minor lower extremity injury - a finding supported by the literature.

\section{Right atrial thrombus and stroke}

- Right-sided mobile thrombi "in transit" from the deep venous system are found in adult case reports or case series in which clots were detected incidentally or during acute pulmonary thromboembolism.

- A recent pediatric literature review article reports in a sum of 122 cases, $91 \%$ of cases to be associated with central venous catheters, $40.8 \%$ in premature neonates, $27.2 \%$ in post cardiac surgery patients, and $19.2 \%$ to have underlying malignancies ${ }^{25}$.

- In our case, the only causative mechanism for the formation of the right-sided thrombus, which was detected upon admission with esophageal ultrasound, was lower extremity deep venous thrombosis, in an otherwise healthy child. A Medline search of Pubmed database using the keywords "right atrial thrombus" and "children or pediatric or paediatric" and "patent foramen ovale" and "stroke" did not reveal any relevant case so, to the best of our knowledge, this is the first documented case of right atrial thrombus resulting from deep venous thrombosis in a pediatric patient with patent foramen ovale and associated ischemic stroke event.

\section{Treatment guidelines}

Young patients with cryptogenic TIA or stroke and PFO should be evaluated for lower-extremity or pelvic venous thrombosis, which would be an indication for anticoagulation. In the setting of a large acute stroke, however, full-dose anticoagulation is not recommended, and an inferior vena cava filter may be the safest alternative. In patients with cryptogenic TIA or stroke, a PFO, and DVT, guidelines from the ACCP currently recommend VKA therapy for 3 months and consideration of PFO closure rather than no VKA therapy or aspirin therapy.

For patients with an ischemic stroke or TIA and both a PFO and a venous source of embolism, anticoagulation is indicated, depending on stroke characteristics (Class I; Level of Evidence A). When anticoagulation is contraindicated, an inferior vena cava filter is reasonable (Class Ila; Level of Evidence C) (New recommendation). 
In cases of concomitant venous and arterial embolism that paradoxical embolism is strongly considered, chronic anticoagulant therapy and an inferior vena cava filter can be justified to prevent further recurrences of both pulmonary and paradoxical embolism ${ }^{26}$.

In the incident of right atrial thrombus, different treatment modalities are reported such as surgical thrombectomy, thrombolysis, anticoagulation therapy or observation only, the choice of which depended mainly on underlying etiology ${ }^{25}$.

In our case, because of the hemorrhagic transformation of the cerebral stroke was an absolute contraindication for the initiation of fibrinolytic therapy, anticoagulation with subcutaneous low molecular weight warfarin along with foramen ovale umbrella placement constituted the selected treatment strategy. This strategy proved to be efficacious during the follow-up period.

\section{Prognosis}

Regarding outcome data, it is referred that permanent moderate-to-severe motor or cognitive disabilities occur in $75-87 \%$ of children with stroke, and death occurs in $5-28 \%{ }^{14}$.

In our case, patient presented with indirect symptoms, such as headache and epileptic fit, these symptoms appearing late from stroke ictus, as seen from the initial MRI presentation of the stroke which was in the hemorrhagic transformation phase and the patient was not on anticoagulation therapy for any reason or didn't report aspirin uptake. Furthermore, she did not suffer any major clinical and neurologic sequelae from the event. These findings, possibly due to the clinically silent anatomical area of the stroke, are contrary to the majority of the cases described in the literature that have unfavorable neurological prognosis.

\section{CONCLUSION}

Cryptogenic AIS is a diagnosis of exclusion. The emergence of cases reporting patients with cryptogenic AIS harboring a patent foramen ovale, tends to reveal the presence of an associated causative factor.

For all the aforementioned reasons, and because many of the aspects of the issue of AIS remain unresolved, we consider that it would be meaningful to present a case that mismatches a lot of aspects of the reported clinical cases and promotes a little known pathophysiologic mechanism, supported by a wide range of clinical and laboratory data.

\section{REFERENCES}

[1] Roach ES, Golomb MR, Adams R, et al. Management of stroke in infants and children: a scientific statement from a Special Writing Group of the American Heart Association Stroke Council and the Council on Cardiovascular Disease in the Young. Stroke. 2008;39:2644-91.

[2] Ganesan V, Prengler M, McShane MA, Wade AM, Kirkham FJ. Investigation of risk factors in children with arterial ischemic stroke. Ann Neurol. 2003;53:167-173.

[3] Kirkham F), Prengler M, Hewes KM, Ganesan V. Risk factors for arterial ischemic stroke in children. J Child Neurol. 2000;15:299-307.

[4] Seminars in thrombosis and hemostasis, volume 29, number 4, 2003.

[5] Alsheikh-Ali Alawi A, Thaler David E, Kent David M. Patent foramen ovale in cryptogenic stroke. Incidental or pathogenic? Stroke Feb. 2009.

[6] Perkovič-Benedik Mirjana, Zaletel Marjan, Pečarič-Meglič Nuška, Podnar Tomaž. A right-to-left shunt and prothrombotic disorders in pediatric patients presenting with transient ischemic attack. Eur J Pediatr. 2013;172:239-245.

[7] Rodriguez Carlos J, Homma Shunichi. Management of patients with stroke and a patent foramen ovale. Current Neurology and Neuroscience Reports. 2004;4:19-22.

[8] Overell JR, Bone I, Lees KR. Interatrial septal abnormalities and stroke A meta-analysis of case-control studies. 2000;55:1172-1179. 
[9] Rodriguez Carlos J, Homma Shunichi. Patent foramen ovale and stroke. Current Treatment Options in Cardiovascular Medicine. 2003;5:233-240.

[10] Perkovič-Benedik Mirjana, Zaletel Marjan, Pečarič-Meglič Nuška, Podnar Tomaž. A right-to-left shunt in children with arterial ischaemic stroke. Arch Dis Child. 2011.

[11] Perkovič Benedik Mirjana, Zaletel Marjan, Pečarič-Meglič Nusčka, Podnar Tomaž. Patent foramen ovale and unexplained ischemic cerebrovascular events in children. Catheterization and Cardiovascular Interventions. 2007;70:999-1007.

[12] Ziesmann Markus T, Monica Nash, Booth Frances A, Rafay Mubeen F. Cardioembolic stroke in children: A clinical presentation and outcome study. Pediatric Neurology. 2014;51:494e502.

[13] Barnes C, DeVeber G. Prothrombotic abnormalities in childhood ischaemic stroke. Thromb Res. 2006;118(1):67-74.

[14] Kirkham F, Sebire G, Steinlin M, Strater R. Arterial ischaemic stroke in children. Review of the literature and strategies for future stroke studies. Thromb Haemost. 2004;92:697-706.

[15] Kenny D, Turner M, Martin R. When to close a patent foramen ovale. Arch Dis Child. 2008;93:255-9.

[16] Di Tullio MR, Sacco RL, Gopal A, et al. Patent foramen ovale as a risk factor for cryptogenic stroke. Ann Intern Med. 1992;117:461-465.

[17] Mallick AA, Ganesan V, O'Callaghan FJ. Mortality from childhood stroke in England and Wales, 1921-2000. Arch Dis Child. 2010;95:12-19.

[18] Ganesan V, Savvy L, Chong WK, Kirkham FJ. Conventional cerebral angiography in the investigation of children with ischaemic stroke. Pediatr Neurol. 1999;20:38-42.

[19] Hill J, Preminger T. Percutaneous PFO closure for paradoxical stroke in 8-kg twins. Catheter Cardiovasc Interv. 2014;84:110e3.

[20] Noser EA, Felberg RA, Alexandrov AV. Thrombolytic therapy in an adolescent ischemic stroke. J Child Neurol. 2001;16:286e8.

[21] Filippi L, Palermo L, Pezzati M, et al. Paradoxical embolism in a preterm infant. Dev Med Child Neurol. 2004; $46: 713 e 6$.

[22] Gunta S, Kamath S. A case of pulmonary embolism and strokein a 16-year-old girl. WMJ. 2012;111:58e60.

[23] Dowling MM, Ikemba CM. Intracardiac shunting and stroke in children: a systematic review. J Child Neurol. 2011;26:72e82.

[24] Perkovič-Benedik M, Zaletel M, Pečarič-Meglič N, et al. A right-to-left shunt and prothrombotic disorders in pediatric patients presenting with transient ischemic attack. Eur J Pediatr. 2013;172:239e345.

[25] Yang JY, Williams S, Brandão LR, Chan AK. Neonatal and childhood right atrial thrombosis: recognition anda risk-stratified treatment approach. Blood Coagul Fibrinolysis. 2010;21(4):301-7.

[26] Perez RD, Maldonado JD, Andresen HM. Acute venous thromboembolic disease and paradoxical embolism. Acta Clin Belg. 2015;70:145e8. 